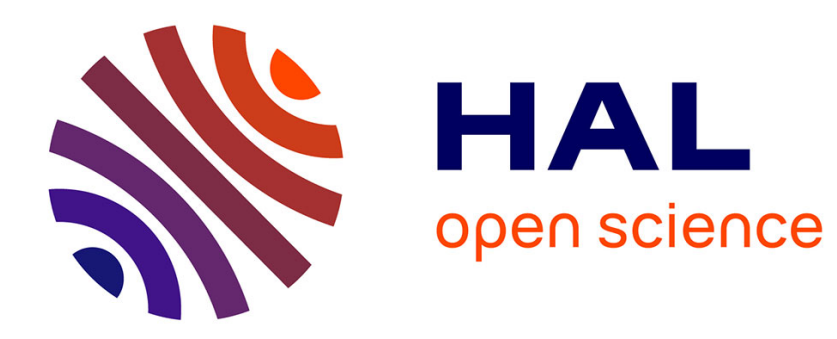

\title{
Les propriétés électroniques et la supraconductivité
}

S. Barišić

\section{To cite this version:}

S. Barišić. Les propriétés électroniques et la supraconductivité. Revue de Physique Appliquée, 1969, 4 (4), pp.545-548. 10.1051/rphysap:0196900404054500 . jpa-00243328

\section{HAL Id: jpa-00243328 https://hal.science/jpa-00243328}

Submitted on 1 Jan 1969

HAL is a multi-disciplinary open access archive for the deposit and dissemination of scientific research documents, whether they are published or not. The documents may come from teaching and research institutions in France or abroad, or from public or private research centers.
L'archive ouverte pluridisciplinaire HAL, est destinée au dépôt et à la diffusion de documents scientifiques de niveau recherche, publiés ou non, émanant des établissements d'enseignement et de recherche français ou étrangers, des laboratoires publics ou privés. 


\title{
LES PROPRIÉTÉS ÉLEGTRONIQUES ET LA SUPRACONDUGTIVITÉ
}

\author{
S. BARIŠ́IĆ $\left({ }^{1}\right)$, \\ Laboratoire de Physique des Solides (2), Faculté des Sciences, 9i-Orsay, France.
}

\begin{abstract}
Résumé. - On discute brièvement les derniers travaux de thermodynamique des supraconducteurs à partir de la théorie microscopique. On insiste davantage sur les systèmes à hautes températures de transition.
\end{abstract}

Abstract. - Some recent achievements in thermodynamics of superconductors, viewed from the microscopic theory, are shortly discussed. Emphasis is put on the systems with high transition temperatures.

1. Les faits expérimentaux [1]. - Une grande quantité de faits expérimentaux a été accumulée pendant la période 1911-1956, alors qu'il n'y avait pas encore de théorie microscopique de la supraconductivité. Il est bientôt apparu que le système électronique subit, pratiquement à lui seul, la transformation de phase, le réseau cristallin et ses vibrations étant peu influencés par la transition.

Le changement de l'énergie interne à 0 oK n'est pas de l'ordre de $k_{\mathrm{B}} T_{\mathrm{c}}$ par électron ( $T_{\mathrm{c}}$, température critique de transition), mais de l'ordre de $\left(k_{\mathrm{B}} T_{\mathrm{c}}\right)^{2} / E_{\mathrm{F}}$, où $E_{\mathrm{F}}$ est l'énergie de Fermi des électrons. Ceci indique que le nombre effectif des électrons, qui abaissent leur énergie de $k_{\mathrm{B}} T_{\mathrm{c}}$, est une petite fraction, $k_{\mathrm{B}} T_{\mathrm{c}} / E_{\mathrm{F}}$, des électrons de conduction. Le principe d'exclusion de Pauli joue donc un rôle important dans la construction de la nouvelle phase.

Une loi d'états correspondants se dégage empiriquement :

- Il existe une échelle réduite sur laquelle la plupart des supraconducteurs se comportent de la même façon.

Exemple : Le champ magnétique thermodynamique suit la loi :

$$
\frac{H_{\mathrm{c}}(T)}{H_{\mathrm{c}}(0)} \simeq 1-\left(\frac{T}{T_{\mathrm{c}}}\right)^{2} \text {. }
$$

2. La théorie B.C.S. - La nature du nouvel ordre, qui s'établit pour $T<T_{\mathrm{c}}$, a été découverte par Cooper [2] : on peut construire, en présence de la mer de Fermi à 0 oK, un nouvel état pour deux élec-

(1) Temporairement détaché de l'Institut de Physique de l'Université de Zagreb, Yougoslavie.

(2) Laboratoire associé au C.N.R.S. trons interagissant par un potentiel attractif, dans lequel les électrons abaissent leur énergie d'une quantité finie, $2 \Delta_{0}$. Cet état est une combinaison linéaire des états $|\mathbf{k} \uparrow 1\rangle$ et $|-\mathbf{k} \downarrow 2\rangle$ où $\mathbf{k}$ est le vecteur d'onde de l'état de Bloch. On prend les spins (dénotés par la flèche) opposés pour éviter la répulsion de Pauli entre les électrons localisés dans l'état de paire.

Par une méthode variationnelle qui utilise l'idée de Cooper, Bardeen, Cooper et Schrieffer ont résolu le problème correspondant à $N$ corps [3]. En particulier, l'énergie de liaison d'une paire à 0 oK est :

$$
2 \Delta_{0} \simeq \hbar \omega_{\mathrm{D}} \mathrm{e}^{-\frac{1}{n_{\mathrm{F}} V}}
$$

$\hbar \omega_{\mathrm{D}}$ est l'énergie de Debye et $V$ le potentiel effectif d'interaction électronique, attribué aux phonons [4]; $n_{\mathrm{F}}$ est la densité d'états électroniques supposée constante dans l'intervalle $\pm \hbar \omega_{\mathrm{D}}$ autour du niveau de Fermi. La constante de couplage $V$ s'ajoute aux deux paramètres $n_{\mathrm{F}}$ et $\omega_{\mathrm{D}}$ qui caractérisent l'état normal. Toutes les propriétés thermodynamiques s'expriment en fonction de ces trois paramètres. Par élimination de ces trois paramètres entre les différentes relations thermodynamiques, on obtient la loi d'états correspondants [3]. Les relations théoriques indépendantes du matériau sont par exemple :

$$
\begin{gathered}
2 \Delta_{0} \simeq 3,5 k_{\mathrm{B}} T_{\mathrm{c}} \\
C_{\mathrm{c}}^{\mathrm{S}}-C_{\mathrm{c}}^{\mathrm{N}} \simeq 1,5 C_{\mathrm{c}}^{\mathrm{N}}
\end{gathered}
$$

$C_{\mathrm{c}}^{\mathrm{s}}$ et $C_{\mathrm{c}}^{\mathrm{N}}$ sont les chaleurs spécifiques électroniques de l'état supraconducteur et normal à $T_{\mathrm{c}}$. Ces relations sont bien vérifiées dans un grand nombre de matériaux $(\mathrm{Sn}, \mathrm{Al} \ldots)$.

3. Les propriétés électroniques dans la détermination de $T_{\mathrm{c}}$. - La température de transition $T_{\mathrm{c}}$ est déterminée par $n_{\mathrm{F}}, \omega_{\mathrm{D}}$ et $V$. Il est très difficile de voir 
dans un matériau réel à quel point $\omega_{\mathrm{D}}$ et $V$ dépendent des propriétés électroniques. En effet, une partie de l'interaction électron-phonon peut être réintégrée dans la redéfinition du spectre des électrons et phonons [5-6]. Le spectre de phonons se trouve ainsi modifié en fonction de propriétés électroniques.

Modèle du jellium : La vitesse du son $s$ est déterminée par la vitesse de Fermi $v_{\mathrm{F}}$ :

$$
s=\left(\frac{m Z}{3 M}\right)^{1 / 2} v_{\mathrm{F}}
$$

$m$ et $M$ sont les masses électronique et ionique, et $Z$ la valence.

Dans ce modèle, la fréquence $\omega_{D}$ est peu différente de la fréquence du plasma ionique, mais dans les métaux polyvalents les fréquences des phonons aux courtes longueurs d'onde sont encore très sensibles à l'écrantage des ions par les électrons (dans les métaux de transition cubique centrés, on trouve expérimentalement $\omega_{D}$ de l'ordre d'un dixième de la fréquence de plasma ionique). Expérimentalement, $\omega_{\mathrm{D}}$ augmente avec la densité d'états $n_{\mathrm{F}}$, mais les calculs sont souvent encore très grossiers.

On se heurte au même genre de difficultés dans le calcul de la constante de couplage. Si l'on néglige tous les effets de retard, c'est-à-dire que l'on suppose que l'échange d'un phonon entre deux électrons est instantané, on trouve dans le modèle du jellium [5] :

$$
n_{\mathrm{F}} V_{\mathrm{p}}=\frac{1}{2+4,7 a_{0}\left|V_{0} Z\right|^{-\mathbf{1} / 3}}
$$

$a_{0}$ est le rayon de Bohr et $V_{0}$ le volume atomique. Bien que grossier, ce résultat contient déjà la caractéristique principale de calculs plus élaborés, à savoir que $n_{\mathrm{F}} V_{\mathrm{p}}$ dépend essentiellement du nombre d'électrons de valence et est pratiquement indépendant de la masse ionique.

Ce résultat peut être testé, en partie, par les mesures d'effet isotopique. En supposant $V=V_{\mathrm{p}}$, on obtient :

$$
\frac{\delta T_{\mathrm{c}}}{T_{\mathrm{c}}}=-\frac{1}{2} \frac{\delta M}{M}
$$

L'effet isotopique observé est en accord avec cette relation, sauf pour les matériaux qui ont des bandes étroites, où il est nettement plus faible. Là, il faut tenir compte de l'interaction de Coulomb à courte portée. La portée de cette interaction est très faible par rapport à la portée de l'interaction due aux phonons, bien que son intensité $V_{\mathrm{c}}$ puisse être forte. On peut introduire une interaction coulombienne effective de portée égale à celle de l'interaction par phonons mais d'intensité $U_{\mathrm{c}}$ plus faible [5-6-7]. Une estimation grossière en est :

$$
U_{\mathrm{c}}=\frac{V_{\mathrm{c}}}{1+n_{\mathrm{F}} V_{\mathrm{c}} \log \frac{\omega_{\mathrm{c}}}{\omega_{\mathrm{D}}}}
$$

où $\omega_{\mathrm{c}}$ définit la vraie portée de l'interaction de Coulomb dans l'espace de phase $\left(\omega_{\mathrm{c}} \gg \omega_{\mathrm{D}}\right)$. L'effet isotopique se trouve maintenant réduit. Avec $V=V_{\mathrm{p}}-U_{\mathrm{c}}$, on obtient :

$$
\frac{\delta T_{\mathrm{c}}}{T_{\mathrm{c}}}=-\frac{1}{2} \frac{\delta M}{M}\left[1-\frac{n_{\mathrm{F}}^{2} V_{\mathrm{c}}^{2}}{1+n_{\mathrm{F}} V_{\mathrm{c}} \log \frac{\omega_{\mathrm{c}}}{\omega_{\mathrm{D}}}}\right] \text {. }
$$

L'énergie $\hbar \omega_{\mathrm{c}}$, étant de l'ordre de l'énergie caractéristique de la bande, est faible dans les bandes étroites, tandis que $n_{\mathrm{F}} V_{\mathrm{c}}$ est fort.

4. Théorie du couplage fort. - Dans les métaux $\mathrm{Pb}$ et $\mathrm{Hg}$ la théorie B.C.S. n'est plus très valable. En particulier, on constate un écart sérieux à la loi des états correspondants.

Le $\mathrm{Pb}$, par exemple, se distingue par une très forte anomalie de Kohn indiquant un fort couplage électronphonon dans l'état normal. Ceci est en accord avec une forte constante de couplage estimée à partir de la transition supraconductrice $\left(n_{\mathrm{F}} V \sim 1\right)$. Ce fort couplage entre les électrons et les phonons empêche $[6,8]$ l'utilisation d'états $|\mathbf{k}\rangle$ comme excitations élémentaires de l'état normal, même dans la petite couche $\pm \hbar \omega_{\mathrm{D}}$, autour du niveau de Fermi, C'est avec ces états, supposés avoir un temps de vie infini, que l'on construit, dans la théorie B.C.S., les états de paires. De plus, la vitesse de phonons étant beaucoup plus petite que celle des électrons au niveau de Fermi ( $\$ 3)$, les effets de retard doivent être pris en ligne de compte.

Eliahsberg et Nambu ont discuté la stabilité de l'état normal dans la limite du couplage fort. La formule approchée pour $T_{\mathrm{c}}$ est due à McMillan [9] :

$$
T_{\mathrm{c}} \simeq \omega_{\mathrm{D}} \exp \left[-\frac{1+\lambda}{\lambda-n_{\mathrm{F}} U_{\mathrm{e}}}\right] \text {. }
$$

Dans la limite de faible couplage $\lambda \ll 1$, on retrouve la formule B.C.S. si $\lambda=n_{\mathrm{F}} V_{\mathrm{p}}$. Cependant, l'expression de l'interaction effective $V_{\mathrm{p}}$ change considérablement quand on inclut les effets de retard. Pour une classe de matériaux (par exemple les matériaux de transition cubique centrés) :

$$
\lambda=\frac{\mathrm{Cte}}{M \omega_{\mathrm{D}}^{2}} .
$$

Contrairement au résultat $\mathrm{du} \oint 3, \lambda$ ne dépend plus $\mathrm{du}$ nombre des électrons de valence mais essentiellement de la fréquence caractéristique des phonons. Cependant, elle ne dépend pas de la masse isotopique $M$; par conséquent, les conclusions concernant l'effet isotopique restent valables.

La température de transition croît quand $\omega_{D}$ décroît à $M$ constant.

Gette conclusion a été vérifiée [10] en comparant $T_{\mathrm{c}}$ des couches très minces (quelques angströms seulement) d'un matériau avec sa valeur pour le matériau massif. Le nombre de liaisons par atome diminue dans une couche très mince et l'effet global est une diminution de $\omega_{\mathrm{D}}$, c'est-à-dire une augmentation de $T_{\mathrm{c}}$. 
Les rapports $\frac{2 \Delta_{0}}{k_{\mathrm{B}} T_{\mathrm{c}}}$ et $\frac{C_{\mathrm{c}}^{\mathrm{S}}-C_{\mathrm{c}}^{\mathrm{N}}}{C_{\mathrm{c}}^{\mathrm{N}}}$ calculés concordent avec les valeurs anormales trouvées (typiquement 4,2 et 2,5$)$.

5. Les composés du type $\mathrm{Nb}_{3} \mathrm{Sn}$. - Dans tous les composés du type $\mathrm{Nb}_{3} \mathrm{Sn}$ à haute $T_{\mathrm{c}}$, examinés jusqu'ici, on observe une anomalie élastique : le module du cisaillement décroît avec la température [11]. Si cette anomalie influence le spectre jusqu'aux phonons à courte longueur d'onde, $\omega_{D}$ est petit, ce qui expliquerait, par les formules de McMillan, la forte $T_{\mathrm{c}}$ dans les composés anormaux et la faible $T_{\mathrm{c}}$ dans les composés du même type mais sans l'anomalie élastique.

Cependant, dans $\mathrm{Nb}_{3} \mathrm{Sn}$ stœechiométrique, la décroissance des constantes élastiques est si forte qu'à 40$50 \mathrm{oK}$ la phase cubique devient instable. Aux températures plus basses, et en particulier à $T_{\mathrm{c}}$, restée pratiquement inchangée, les constantes élastiques de la phase tétragonale retrouvent l'ordre de grandeur qu'elles avaient à haute température [12].

La constante de couplage est donc pratiquement indépendante de l'existence de l'anomalie élastique, ce qui indique que cette anomalie concerne uniquement les grandes longueurs d'onde.

Une théorie convaincante des composés du type $\mathrm{Nb}_{3} \mathrm{Sn}$ doit expliquer les fortes variations de $T_{\mathrm{c}}$ parmi les différents composés, la constance de $T_{\mathrm{c}}$ dans les différents échantillons d'un même composé, tout en supposant la fréquence caractéristique des phonons, concernés par la supraconductivité, comme un paramètre peu variable.

Le modèle de Labbé, Friedel et Barišić permet de comprendre l'essentiel des phénomènes décrits.

Dans ce modèle, les électrons $d$ sont fortement liés aux chaînes denses d'atomes de transition. Ceci se traduit par une structure très fine de la densité d'états, avec des pics très dégénérés aux extrémités des trois bandes $d$. L'anomalie élastique s'explique si on suppose que le niveau de Fermi est très près d'un des pics $[13,14]$ ( fig. 1).

Dans l'état supraconducteur, la plupart du petit nombre de porteurs reste dans le pic près du niveau de Fermi, dans la région où le concept des quasiparticules est très vraisemblablement justifié. Donc il suffit d'adapter la théorie B.C.S. à la structure fine de la densité d'états $[15,16]$.

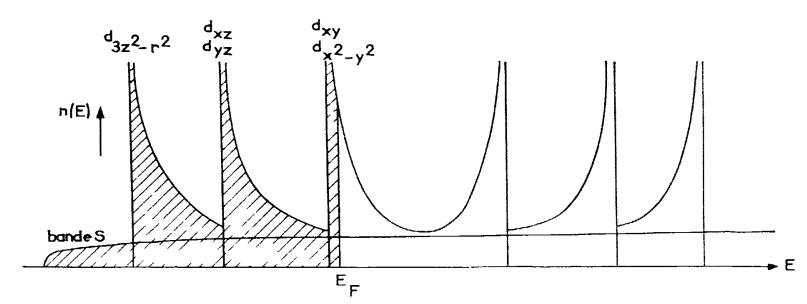

FIG. 1.

A $V$ constant, la température de transition passe par un large maximum en fonction du nombre de porteurs. Dans cette région, $T_{\mathrm{c}}$ dépend de $\omega_{\mathrm{D}}$ uniquement par $V$ du fait que ce sont les états de pic, près du niveau de Fermi, qui donnent la contribution prépondérante à $T_{\mathrm{c}}$. Ceci explique, d'une part, l'absence presque complète d'effet isotopique observée dans $\mathrm{Nb}_{3} \mathrm{Sn}$ et, d'autre part, le peu d'influence de la transition martensitique sur la valeur de $T_{\mathrm{c}}$. En effet, cette transition fait varier le nombre de porteurs dans le pic, mais on reste dans la région du maximum de $T_{\mathrm{c}}$.

La constante de couplage estimée est plutôt inférieure aux valeurs habituelles : $V \simeq 0,1 \mathrm{eV} /$ at.

Quand le niveau de Fermi est loin d'un pic, $n_{\mathrm{F}} V$ est faible, et par une formule B.C.S. on trouve une faible température de transition accompagnée de l'effet isotopique.

Un cas intermédiaire est $\mathrm{Mo}_{3} \mathrm{Ir}$ avec $T_{\mathrm{c}}=7{ }^{\circ} \mathrm{K}$ et $\frac{\delta T_{\mathrm{c}}}{T_{\mathrm{c}}} \simeq-0,3 \frac{\delta M}{M}$.

Conclusion. - Si la nature du phénomène de supraconductivité est bien comprise, aujourd'hui on est encore loin de pouvoir « calculer » un supraconducteur fort. Si on peut dire parfois qu'un matériau ne sera pas supraconducteur, on n'a pas encore trouvé une règle empirique indiquant les supraconducteurs et surtout les supraconducteurs forts.

Deux voies semblent cependant intéressantes à prospecter : les composés intermétalliques ou les systèmes organiques quasi unidimensionnels, et les structures isotropes à $\omega_{\mathrm{D}}$ petit.

Remerciements. - L'auteur a bénéficié de discussions utiles avec M. le Professeur J. Friedel et le Docteur J. Labbé. Il remercie les organisateurs de la conférence d'Aussois pour leur invitation.

\section{BIBLIOGRAPHIE}

[1] LynTon (E. A.), Superconductivity, London, New York, Wiley \& Sons, 1962.

[2] CoOper (L. N.), Phys. Rev., 1956, 104, 1189.

[3] BARdeEn (J.), CoOper (L. N.) et SchriefFER (J. R.), Phys. Rev., 1957, 108, 1175.

[4] Fröhlich (H.), Proc. Roy. Soc. (London), 1950, A 215, 291.
[5] DE Gennes (P. G.), Superconductivity of Metals and Alloys, Benjamin, N.Y.-Amsterdam, 1966.

[6] SCHRIEFFER (J. R.), Theory of Superconductivity, Benjamin, 1966.

[7] Garland Jr (J. W.), Phys. Rev. Letters, 1963, 11, 114.

[8] Scalapino (D. J.), Schrieffer (J. R.) et WiLkINS (J. W.), Phys. Rev., 1966, 148, 263. 
[9] MCMILLAN (W. L.), Phys. Rev., 1968, 167, 331.

[10- Strongin (M.), Kammerer (O. F.), Crow (J. E.), PARKS (R. D.), DOUgLass Jr (D. H.) et JENSEN (M. A.), Phys. Rev. Letters, 1968, 21, 1320.

[11] TESTARDI (L. R.), SODEN (R. R.), GREINER (E. S.), Wernick (J. H.) et Chirba (V. G.), Phys. Rev., 1967, 154, 389.

[12. ReHWALD (W.), Phys. Letters, 1968, 27 A, 287.
[13] Labbé (J.) et FrifodeI (J.), J. Physique, 1966, 27, 153, 303 .

[14] BARIšIć (S.) et LabBÉ (J.), J.P.C.S., 1967, 28, 2477.

[15] Labbé (J.), Barišić (S.) et Friedel (J.), Phys. Rev. Letters, 1967, 19, 1039.

[16] Bonnerot' (J.), Hallais (J.), Barišić (S.) et LabBé (J.), J. Physique, 1969, 30, 701. 